\title{
A importância do farmacêutico na gestão dos medicamentos nas Estruturas Residenciais para Pessoas Idosas em Portugal
}

\author{
Pharmacists and medication management in senior care \\ facilities in Portugal
}

Recebido em: 10/02/2021 Aceito em: 20/10/2021
Victoria BELL; João Rui PITA Faculdade de Farmácia, Universidade de Coimbra, Polo das Ciências da Saúde. Azinhaga de Santa Comba. CEP 3000-548. Coimbra, Portugal.

E-mail:victoriabell@ff.uc.pt

\section{ABSTRACT}

Population aging has changed society and is challenging health professionals. Institutionalization of the elderly is frequently the only alternative to safeguard their health and to ensure their quality of life. Chronic diseases, prevalent in these citizens, are mostly controllable with medicines. Medication management in senior care facilities must ensure that regulatory guidelines on the quality and effectiveness of medicinal products are ensured. Pharmacists as qualified and legally acknowledged health professionals to perform these functions should be included in the multidisciplinary teams responsible for providing health care and well-being to the institutionalized elderly.

Keywords: medicine management; senior care facilities; pharmacist.

\section{RESUMO}

O crescente envelhecimento da população tem suscitado mudanças na sociedade e tem constituído um desafio constante para os profissionais de saúde. A institucionalização do idoso surge, muitas vezes, como a alternativa mais viável para salvaguardar a sua saúde e zelar pela sua qualidade de vida. As doenças crónicas não transmissíveis, prevalentes em geriatria, podem ser atenuadas por mudanças nos hábitos de vida, mas são maioritariamente controláveis através de medicamentos. A gestão dos medicamentos nas estruturas residenciais para pessoas idosas, visando a promoção de um envelhecer saudável, tem de assegurar que as diretrizes regulamentares referentes à qualidade e à eficácia dos mesmos esteja garantida. $\mathrm{O}$ farmacêutico apresenta-se como o profissional de saúde mais habilitado e legalmente reconhecido para exercer estas funções. Como tal, os farmacêuticos devem ser incluídos na equipa multidisciplinar de profissionais que diariamente colaboram para manter a saúde e proporcionar bem-estar ao idoso institucionalizado, bem como na prestação de cuidados paliativos aos pacientes portadores de doenças sem possibilidade de cura.

Palavras-chave: medicamentos; gestão; lares para idosos; farmacêutico. 


\section{INTRODUÇÃO}

O envelhecimento da população é um dos maiores sucessos da humanidade; contudo, é também um dos seus maiores desafios devido às consequências sociais, econômicas e políticas a ela inerentes. Só o empenho individual e coletivo permitirá que o idoso permaneça saudável, ativo e autónomo o maior tempo possível.

As novas realidades sociais, os novos problemas da sociedade decorrentes do envelhecimento da população, das alterações demográficas, do surgimento de novas doenças, da passagem a doenças crônicas de muitas doenças anteriormente incuráveis suscitaram novos e modernos desafios terapêuticos (1).

Nos últimos anos, o alargamento do arsenal terapêutico no campo do diagnóstico, da prevenção e do tratamento de doenças muito diversificadas (e.g. demências, a hipertensão arterial, o diabetes e a osteoporose) cruza-se com uma vasta panóplia de dispositivos tecnológicos médicos e de outros produtos de saúde. A investigação tanto em medicamentos como em produtos e suplementos de saúde é muito vasta, incluindo fortificação de alimentos $(2,3)$. A sua finalidade última é o doente e o cidadão em geral, a sua saúde e o seu bem-estar.

O envelhecimento da população - fenômeno natural, irreversível e com impacto em toda a sociedade - e a eventual necessidade de suporte social, por meio da institucionalização da pessoa idosa, exige uma responsabilidade coletiva, e representa um desafio constante para os profissionais de saúde. A prestação de cuidados de saúde de qualidade, de modo a promover um envelhecimento ativo e a interação social do idoso institucionalizado, requer profissionais competentes, que permitam responder não só às necessidades individuais dos utentes, mas também contribuir para a melhoria da saúde da sociedade(4).

De forma a melhorar ou a manter o seu bem-estar físico, mental e social, é fundamental que o idoso, mesmo institucionalizado, se envolva na vida social, cultural, espiritual e civil e que permaneça fisicamente ativo. Sendo o envelhecimento um processo lento onde estão implícitas alterações biológicos, psicológicos e sociais, são diversos os tipos de profissão que devem acompanhar o idoso.

Neste contexto restringimo-nos ao papel dos farmacêuticos, reconhecendo que deve ser uma atividade programada de forma multidisciplinar envolvendo também enfermeiros, médicos, psicólogos, fisioterapeutas, bombeiros, auxiliares entre outros. Os farmacêuticos têm nesta articulação um papel fundamental. Os medicamentos e os produtos de saúde são, sujeitos a uma malha jurídica e regulamentar muito restrita. Os diferentes protagonistas, farmacêuticos e outros, devem cumprir leis e regulamentos, e uma apertada malha de normas éticas.

No presente artigo abordamos a importância da aquisição, do armazenamento, da distribuição e da cedência de medicamentos nas Estruturas Residenciais para Pessoas Idosas. Destacamos as vantagens que, na nossa opinião, advêm da intervenção de profissionais de saúde qualificados nessas tarefas e apresentamos algumas lacunas que, em nosso entender, existem neste sector e no atual sistema de funcionamento destas entidades. São tecidas algumas considerações sobre as vantagens do acompanhamento farmacêutico em doentes idosos institucionalizados e apresentadas algumas vantagens que resultariam do compromisso de pessoal farmacêutico para a gestão dos medicamentos nestas instituições.

\section{O CIDADÃO IDOSO}

O envelhecimento da população é uma realidade incontestável com a qual temos sido confrontados nas últimas décadas (5).

Alcançar um envelhecimento saudável é uma tarefa na qual a literacia em saúde do utente exerce um papel preponderante. O conhecimento da sua saúde e a melhor forma de a gerir, assim como compreender o modo de funcionamento do sistema de saúde são fundamentais para que o utente consiga manter a sua qualidade de vida e o seu bem-estar à medida que envelhece (6).

É importante antever o envelhecimento promovendo a saúde ao longo da vida por meio de atitudes preventivas. Uma alimentação saudável, a prática regular de exercício físico, a realização de testes preditivos de algumas das doenças crônicas 
mais prevalentes podem contribuir para um envelhecimento mais saudável, com maior independência, autonomia e bem-estar (7).

Com o envelhecimento surgem alterações fisiológicas, acompanhadas muitas vezes por transformações sociais e por algumas fragilidades econômicas (8). Estas situações podem condicionar a alimentação do idoso e comprometer o aporte de alguns nutrientes essenciais. Com o avançar da idade as necessidades energéticas diminuem, existem alterações na dentição (9) e na capacidade de absorção intestinal. Muitos idosos vivem sozinhos, as suas aptidões físicas e a sua mobilidade encontram-se reduzidas (10) dificultando a aquisição de alimentos, que os leva frequentemente a optar por dietas monótonas que podem deteriorar o seu estado de saúde (11). A falta de interação social com familiares e amigos pode ter um impacto negativo na saúde mental do idoso (12), agravando o seu isolamento e potenciando a ocorrência de estados depressivos (13).

$\mathrm{Na}$ sua vasta maioria, os idosos, são doentes com doenças crónicas, polimedicados (14) e que evidenciam, por vezes, problemas de adesão à terapêutica. A polimedicação apesar de necessária para o controle das respetivas doenças, gera, frequentemente, situações que podem pôr em risco a saúde do idoso (15). A utilização correta e atempada da medicação, a conservação dos medicamentos de acordo com as condições de temperatura e de umidade exigidas e o acompanhamento regular do doente idoso são cuidados simples, mas sem dúvida indispensáveis para assegurar a sua saúde e o seu bem-estar (16).

Apesar das diferenças educacionais, culturais e socioeconómicas e do seu nível de independência, os idosos apresentam-se como uma faixa etária com problemas convergentes.

Por vezes a autonomia do idoso fica comprometida e ele já não consegue realizar, sem apoio, as tarefas diárias básicas, comprometendo a sua saúde e mesmo a sua segurança. A estrutura familiar que servia de apoio tanto para as gerações mais novas como para as gerações mais velhas tem-se transformado. As alterações sofridas nesta estrutura e as modificações no seu funcionamento criaram lacunas na rede de apoio social às quais foi necessário dar resposta (8). Vários desafios decorrentes destas alterações têm sido alvo de reflexão e implementação de medidas com o objetivo de promover o bem-estar dos idosos.

Ações multidisciplinares, sustentadas em bases legais, têm tido como prioridade a prevenção da doença e a promoção da saúde; no entanto, existem ainda muitos idosos cuja qualidade de vida está aquém do desejável (5).

Atualmente, existem em Portugal várias respostas sociais para o acompanhamento de pessoas idosas. Destas, destacam-se as seguintes: Serviço de apoio domiciliários (SAD), Centro de convívio, Centro de dia, Centro de noite, Acolhimento familiar para pessoas idosas e adultas com deficiências, Cuidador informal e Estrutura residencial para pessoas idosas. Excetuando as Estruturas Residenciais para Pessoas Idosas, todas as outras respostas sociais disponíveis atuam de modo a promover a autonomia e a evitar a institucionalização do idoso. Todavia, a institucionalização, apresenta-se, muitas vezes, como a única forma dos familiares conseguirem providenciar um acompanhamento que responda às necessidades singulares daqueles cidadãos.

A Portaria n. ${ }^{\circ}$ 67/2012, de 21 de março, "define as condições de organização, funcionamento e instalação a que devem obedecer às estruturas residenciais para pessoas idosas". O referido diploma legal define estrutura residencial para pessoas idosas como um "estabelecimento para alojamento coletivo, de utilização temporária ou permanente, em que sejam desenvolvidas atividades de apoio social e prestados cuidados de enfermagem"(17). Nos serviços prestados nestes estabelecimentos incluem-se o acesso a cuidados de saúde e a administração de medicamentos.

Apesar do funcionamento destes estabelecimentos se encontrar devidamente regulamentado, existem questões determinantes relacionadas com o armazenamento, distribuição, dispensação e administração dos medicamentos que, na nossa opinião, ainda não foram convenientemente abordadas e que poderão ter repercussões na saúde dos idosos acolhidos.

\section{O MEDICAMENTO E O SEU MARCO LEGAL}

O medicamento é um produto decorrente de uma longa investigação científica, produzido de acordo com adequadas normas técnicas e sujeito 
a um apertado espartilho de qualidade. Tem finalidades clínicas (de diagnóstico, de prevenção e de tratamento) e é subordinado a balizas jurídicas e regulamentares. Está sujeito às normas do mercado e constitui uma preocupação econômica para o consumidor, para as organizações que coparticipam e para o Estado. O medicamento deve ser utilizado de forma racional assegurando que cada doente receba a medicação certa, em tempo certo, que a utilize corretamente e que dela retire o maior benefício possível (18).

A prescrição do medicamento é da responsabilidade do médico assistente (19) e, de acordo com a legislação vigente em Portugal, a sua venda só pode ser efetuada em locais de dispensa de medicamentos autorizados, sob a supervisão de um farmacêutico, no caso das farmácias, e de um farmacêutico ou de um técnico de farmácia no caso dos locais de venda de medicamento não sujeitos a receita médica (20). Ou seja, os medicamentos sujeitos a receita médica (MSRM), os medicamentos sujeitos a receita médica de dispensa exclusiva em farmácia (MSRM-EF) e os medicamentos não sujeitos a receita médica de dispensa exclusiva em farmácia (MNSRM-EF), só podem ser vendidos em farmácias. Os medicamentos não sujeitos a receita médica (MNSRM) podem ser adquiridos em farmácias e em locais de venda de medicamentos não sujeitos a receita médica. Esta premissa legal tem como objetivo promover o "uso racional do medicamento, no interesse dos doentes e da saúde pública"(20).

A forte regulamentação do medicamento em todas as suas vertentes assegura que a sua qualidade, a sua eficácia e a sua segurança sejam garantidas. Em Portugal, o Decreto-lei n. ${ }^{\circ} 176 / 2006$, de 30 de agosto, estabelece o regime jurídico dos medicamentos de uso humano (20). Este diploma institui as normas legais a que devem obedecer a autorização de introdução no mercado, a manufatura, a importação, a exportação, a comercialização, a rotulagem e informação, a publicidade, a distribuição, a farmacovigilância e a utilização dos medicamentos para uso humano. Desde a sua publicação foram feitas 12 alterações a este diploma legal, sendo a mais recente o Decreto-lei n. ${ }^{\circ} 112 / 2019$, de 16 de agosto (21). Todas as alterações foram efetuadas no sentido de garantir uma resposta legal adequa- da aos constantes desafios que surgem na área do medicamento. O diploma anteriormente mencionado, refere que "a utilização dos medicamentos no âmbito do sistema de saúde, nomeadamente através da prescrição médica ou da dispensa pelo farmacêutico, deve realizar-se no respeito pelo princípio do uso racional do medicamento, no interesse dos doentes e da saúde pública" e que "os profissionais de saúde assumem, no âmbito das respetivas responsabilidades, um papel fundamental na utilização racional dos medicamentos e na informação dos doentes e consumidores quanto ao seu papel no uso correto e adequado dos medicamentos".

Para a implementação do uso racional dos medicamentos é, contudo, necessário desenvolver estratégias adequadas (22). A seleção de medicamentos, a construção de formulários terapêuticos, a gestão adequada dos serviços farmacêuticos, a dispensa e o uso apropriado de medicamentos, a farmacovigilância, a educação dos utentes quanto aos riscos da automedicação, da interrupção e da troca de medicamentos prescritos, são exemplos.

A enorme multiplicidade de produtos farmacêuticos, os limites e os desafios impostos pelas diferentes formas de uso do medicamento e até a influência da indústria farmacêutica podem impactar fortemente no uso racional dos medicamentos.

O regime jurídico das farmácias de oficina (23), também denominadas muitas vezes como farmácias comunitárias, estabelece que a venda de medicamentos ao público só pode ser feita em farmácias e em locais de venda de medicamentos não sujeitos a receita médica. $\mathrm{O}$ referido diploma também determina que entrega desses mesmos medicamentos ao público só pode ser efetuada por pessoal dos mencionados estabelecimentos legalmente habilitado. Estas restrições legais foram implementadas de modo a garantir a proteção da saúde pública e assegurar que o medicamento seja utilizado de forma racional.

Por outro lado, estipula ainda que todas as pessoas que trabalhem numa farmácia são "obrigadas a guardar segredo dos factos que tenham conhecimento em razão da sua atividade", garantindo deste modo a confidencialidade e privacidade do utente. As farmácias também devem ter mecanismos que assegurem a segurança dos medicamentos e um sistema de medição e registo da 
temperatura e da humidade que garanta a correta conservação dos mesmos.

A Portaria n. ${ }^{\circ} 1427 / 2007$, de 2 de novembro, regula as condições e os requisitos da dispensa de medicamentos ao domicílio e por meio da Internet (24). De acordo com este documento legal, a entrega de medicamentos, por uma farmácia, deve ser sempre supervisionada por um farmacêutico. No momento da entrega dos referidos medicamentos, o diretor técnico deve assegurar que é transmitida toda a informação necessária à sua correta utilização.

A Portaria n. ${ }^{\circ}$ 1429/2007, de 2 de novembro, define os serviços farmacêuticos que podem ser prestados pelas farmácias (25). Entre estes serviços encontram-se a "administração de medicamentos" e os "programas de adesão à terapêutica, de reconciliação da terapêutica e de preparação individualizada de medicamentos". O mesmo diploma determina ainda que as farmácias podem "promover campanhas e programas de literacia em saúde, prevenção da doença e de promoção de estilos de vida saudáveis" e que os serviços mencionados deverão ser prestados por profissionais legalmente habilitados.

\section{O PAPEL DO FARMACÊUTICO}

Enquanto profissionais de saúde, é bem evidente o papel relevante dos farmacêuticos tanto no Sistema como nos Serviços de Saúde e na saúde da população. São detentores de competências específicas para interferir e direcionar para a melhor utilização do medicamento e para instigar ao seu uso racional sobretudo na gestão do circuito do medicamento nos cuidados de saúde primários, na operacionalização das políticas de saúde, na informação sobre medicamentos e tecnologias de informação ou nas atividades de farmácia clínica, cuidados farmacêuticos e farmacoterapia (26).

O farmacêutico pode e deve ainda atuar na interface entre administrações regionais e a rede privada de farmácias comunitárias e os cuidados de saúde primários, os cuidados hospitalares, os cuidados continuados ou paliativos (27). No seu papel como educador, promove o aumento da literacia em saúde da população e contribui para a diminuição das desigualdades em saúde da mesma (28).
Os constantes desafios colocados ao farmacêutico por uma sociedade cada vez mais informada exigem que este, enquanto profissional, aperfeiçoe as suas competências e se mantenha ao corrente das novidades científicas que a cada momento vão surgindo (29). O rigoroso cumprimento dos princípios éticos e deontológicos, a responsabilidade, a competência, a independência das práticas científicas e profissionais assim como a visualização dos problemas sob a ótica do doente ou utente são fatores que caracterizam os farmacêuticos (30).

Devido ao seu posicionamento ímpar como especialista do medicamento e em saúde pública encontra-se diretamente implicado no bem-estar da população em geral e do doente em particular (31). O medicamento, núcleo da atividade do farmacêutico, é um produto complexo do ponto de vista técnico e científico, com legislação complexa, a cada momento atualizada, e sujeito a normas éticas e deontológicas.

Os produtos de saúde, tal como hoje os conhecemos, do ponto de vista de variedade, de aplicação e de normas jurídicas, constituem hoje um forte desafio ao farmacêutico moderno. $\mathrm{O}$ farmacêutico tem acompanhado as alterações científicas, sociais e culturais que surgiram e surgem, integrando novos saberes e adaptando-se às novas realidades de modo a ir ao encontro das necessidades daqueles que servia e serve (32).

A importância do farmacêutico como educador em saúde apresenta-se como uma necessidade crescente numa sociedade cada vez mais confrontada com novidades permanentes no setor da saúde (33). Novos medicamentos, assim como formas inovadoras de os administrar surgem com enorme cadência. Os utentes necessitam de ser informados/formados sobre a sua utilidade terapêutica e a forma correta de os utilizar.

Na transição do século XX para o século XXI operaram-se alterações profundas, tanto nos planos ético-jurídico, científico, técnico e econômico, mas também nos planos profissional, institucional e organizacional. Portugal não tem sido exceção e representa bem essas alterações que se têm verificado no plano da farmácia e do medicamento, tanto ao nível dos produtos, como no plano das instituições, mas também no campo científico e tecnológico. 
ESTRUTURAS RESIDENCIAIS PARA PESSOAS IDOSAS (ERPI)

Em Portugal, entre 1998 e 2018, verificou-se um aumento de $105 \%$ no número de estruturas residenciais para pessoas idosas (34). Em 2018, segundo dados do Gabinete de Estratégia e Planeamento, existiam em Portugal 2507 ERPI, com capacidade para 100518 utentes (35).

A institucionalização suscita uma profunda mudança na vida do idoso. Há uma ruptura com o seu espaço e com as suas relações. Os seus hábitos diários são alterados, sendo necessário ajustar a sua privacidade e a sua individualidade a uma vivência conjunta. Esta transição, por vezes, não decorre de forma equilibrada e a adaptação do idoso a este novo ambiente nem sempre é tranquila (36).

Os utentes das ERPI provêm de vivências muito heterogéneas, porém são maioritariamente doentes crônicos, polimedicados (37) e predominantemente sem a autonomia necessária para gerir a sua medicação.

Por este motivo as ERPI têm de assegurar a aquisição, o armazenamento e a distribuição dos medicamentos prescritos, assim como garantir a administração correta dos mesmos aos doentes. Somos da opinião que a intervenção do farmacêutico é indispensável neste processo.

Em Portugal, de acordo com a legislação vigente, é obrigatória a presença de um farmacêutico responsável em todo o circuito do medicamento. Os responsáveis técnicos das indústrias produtoras de medicamentos, dos armazéns de distribuição de medicamentos e das farmácias têm de ser farmacêuticos. São vários os documentos (38) e diplomas legais $(19,39)$ que consideram a atividade farmacêutica essencial para garantir a defesa e a proteção da saúde, a satisfação das necessidades da população, assim como a racionalização do consumo de medicamentos.

Apesar de devidamente regulamentadas, as estruturas residenciais para pessoas idosas não têm qualquer obrigatoriedade legal de possuírem um serviço farmacêutico. No nosso entender. esta lacuna legal constitui um motivo de preocupação. A inclusão do farmacêutico na equipe multidisciplinar que integra as ERPI, mesmo em tempo não integral, traria melhorias significativas ao sistema de gestão de medicamentos destas instituições, com resultados positivos para a saúde e para o bem-estar dos residentes institucionalizados. Ao assumir a gestão dos medicamentos nas ERPI, o farmacêutico seria responsável pela aquisição, pelo armazenamento e pela distribuição dos medicamentos, assim como pela administração correta dos mesmos.

Os conhecimentos e a formação deste profissional de saúde são garantia que todas as normas legais sejam implementadas e cumpridas. No entanto, a intervenção do farmacêutico nas ERPI pode ser muito mais abrangente. O farmacêutico é o profissional de saúde mais indicado para avaliar os resultados da utilização dos medicamentos. A sua intervenção é importante para a redução de erros, de interações e de problemas relacionados com os medicamentos, contribuindo para uma melhor e mais racional utilização dos mesmos (40).

Em 2014, o Instituto Português da Qualidade (IPQ) por meio da Comissão Sectorial para a Saúde CS/09, emitiu uma recomendação para a "Gestão da Medicação nas Estruturas Residenciais para Pessoas Idosas (ERPI)"(41). Nesse documento a referida comissão reconhece que em Portugal "é evidente a escassez de legislação específica que defina procedimentos e normas de qualidade, em relação à prescrição, aquisição, armazenamento, distribuição, administração e utilização dos medicamentos nas ERPI"(41) e que os idosos institucionalizados devem usufruir das mesmas garantias de qualidade, no que concerne à utilização dos medicamentos, que os utentes da comunidade (37).

Na opinião dessa comissão, seria vantajosa a participação da Rede de Farmácias no circuito do medicamento nas ERPI. A instituição de procedimentos e a correta atribuição de responsabilidades no circuito dos medicamentos é, de acordo com a referida comissão, fundamental para que os doentes "recebam os seus medicamentos de forma correta, efetiva e segura" (41). Deste modo, os intervenientes no circuito do medicamento devem ter "competências na gestão do medicamento, dentro das suas atribuições profissionais, por forma a garantir que os doentes usufruam de um benefício terapêutico máximo, resultante do tratamento com medicamentos" (41). 
Relativamente à aquisição e dispensa de medicamentos, a comissão recomenda que "deve ser assegurado que um profissional legalmente habilitado avalie a prescrição dos doentes e preste toda a informação necessária ao uso correto, efetivo e seguro do medicamento, com vista a prevenir resultados negativos associados à medicação" (41). Sempre que haja a admissão de um novo doente deverá ser feita a reconciliação terapêutica entre a medicação anteriormente tomada e a prescrita na instituição. Para cada utente deve ser elaborado um registo completo e atualizado da sua medicação. Estes procedimentos reduzem a ocorrência de erros e contribuem para a saúde e bem-estar do doente (42).

O armazenamento e a conservação dos medicamentos devem, ainda, garantir que sejam asseguradas todas as condições legalmente exigidas. A zona de armazenamento deve ser reservada a profissionais de saúde autorizados e para todos os medicamentos deve ser feito o registo do lote e do respetivo prazo de validade. As condições de temperatura e umidade devem ser controladas e registadas e adequadas às especificações de cada medicamento. O sistema de distribuição de medicamentos instituído deve garantir que o doente certo, receba o medicamento certo, na dose certa, através da via de administração certa e na hora certa. Para avaliar a necessidade, a efetividade e a segurança da terapêutica instituída, esta deve ser monitorizada periodicamente e revista pelo menos duas vezes por ano. Os profissionais de saúde devem promover a adesão à terapêutica e registar as falhas da mesma. Devem igualmente registar as interações, os efeitos adversos e os problemas relacionados com os medicamentos, notificando todas as situações que julguem necessárias (41).

O papel do farmacêutico na gestão dos medicamentos dos doentes já é reconhecido em diversos países. Nos Estados Unidos da América (EUA) (43), no Reino Unido (44)(45),(46), na Austrália (47) e no Canadá (48), sendo que esta função do farmacêutico está claramente identificada e aceita como necessária. O farmacêutico que presta serviços especializados de assistência a pessoas idosas ou a doentes é designado de Consultant Pharmacist (Farmacêutico consultor).
Recentemente nos EUA, a American Society of Consultant Pharmacists (ASCP), tem optado pela designação de Senior Care Pharmacist, para os farmacêuticos especializados no apoio a doentes idosos (49). Esta associação, fundada em 1969, congrega farmacêuticos consultores com intervenção em diversos tipos de instituições, como ERPI, centros de dia, serviços domiciliários, entre outros (50).

No Reino Unido, em 2003, foi publicado o documento "A Vision for Pharmacy in the New NHS" (51). Este documento reconhece a importância do farmacêutico como educador em saúde e na promoção no uso adequado e racional do medicamento. Refere igualmente que este profissional de saúde detém um elevado nível de confiança na sociedade, reconhecendo e valorizando as suas competências técnicas e científicas.

Para fazer face às exigências crescentes da sociedade na área da saúde, o farmacêutico teve de se adaptar e disponibilizar novos serviços de modo a ir ao encontro das necessidades da população. O apoio na gestão de medicamentos em doentes com pouca autonomia, a revisão periódica da medicação em doentes polimedicados e a monitorização de doentes são alguns exemplos destes serviços. A sua implementação tem contribuído para melhorar a saúde da população e ajudar a reduzir as desigualdades em saúde (52).

$\mathrm{O}$ "Guidance for the Development of Consultant Pharmacist Posts" (53) reconhece a necessidade da criação de Farmacêuticos consultores no Reino Unido e define as regras para a criação desta nova categoria profissional. São apresentados como características principais do Farmacêutico consultor o conhecimento especializado, a capacidade de desenvolver estratégias para a implementação de serviços de gestão da medicação, de contribuir para a formação dos profissionais de saúde, de integrar equipas multiprofissionais e ter capacidade de liderança (53). Em 2020, o documento foi atualizado por meio da publicação do "Consultant Pharmacist Guidance" (54).

Nos EUA, a regulamentação federal determina que os lares têm de possuir serviços farmacêuticos permanentes, sob a responsabilidade de um farmacêutico, que assegurem a gestão dos medicamentos. Esta imposição legal tem como 
objetivo proteger a saúde e zelar pela segurança dos doentes institucionalizados (55). As vantagens da intervenção de um farmacêutico na gestão dos medicamentos e na medicação de doentes institucionalizados estão estudadas (47) e são reconhecidas, naquele país, há mais de 40 anos (43).

\section{CONCLUSÃO}

Envelhecer com saúde, permanecendo autônomo e independente o mais tempo possível, requer empenho individual e coletivo e é, atualmente, um desafio para os profissionais de saúde.

\section{REFERÊNCIAS}

1. Shakoori IS, Aslam F, Ashraf G, Akram H. Understanding chronic disease risk factors and multimorbidity. Int J Community Med Public Heal. 2020 Apr 24;7(5):1990-3. DOI: 10.18203/2394-6040.IJCMPH20201556

2. Bell V, Ferrão J, Fernandes T. Nutritional Guidelines and Fermented Food Frameworks. Foods [Internet]. 2017;6(8):65. DOI: 10.3390/foods6080065

3. Bell V, Barros AB, Fernandes TH. Food Fortification in Sub Saharan Africa: Science or Business? In: Fernandes TH, Ferrão J, Facknath S, editors. Food and Nutrition Security in Africa. Mozambique: Alcance Ed.; 2020.

4. WHO. Envelhecimento ativo: uma política de saúde [Internet]. 2005. Available from: http://www.dgeep.mtss. gov.pt.

5. PORTUGAL. Estratégia Nacional para o Envelhecimento Ativo e Saudável - 2017-2025. Grupo de Trabalho Interministerial (Despacho n.o12427/2016). Direção Geral da Saúde. 2017. Available from: https://www.sns.gov.pt/ wp-content/uploads/2017/07/ENEAS.pdf.

6. PORTUGAL. Plano de ação para a literacia em saúde 2019-2021. Direção de Serviços de Prevenção da Doença e Promoção da Saúde (DSPDPS) Divisão de Literacia S e B-E. PORTUGAL. Direção Geral da Saúde. Lisboa; 2019. Available from: https://www.dgs.pt/em-destaque/ programa-nacional-de-educacao-para-a-saude-literacia-eautocuidados.aspx

7. PORTUGAL. Plano nacional de saúde: Revisão e extensão a 2020. Direção-Geral da Saúde. Lisboa; 2015. Available from: https://bicsp.min-saude.pt/pt/biufs/O QUE OFERECEMOS/Plano-Nacional-de-Saude-Revisao-eExtensao-a-2020.pdf.pdf.

8. Paskaleva D, Tufkova S. Social and Medical Problems of the Elderly. J Gerontol Geriatr Res. 2017; 6(3):3-7. DOI: 10.4172/2167-7182.1000431

9. Masood M, Newton T, Bakri NN, Khalid T, Masood Y. The relationship between oral health and oral health related quality of life among elderly people in United Kingdom. J Dent [Internet]. 2017;56:78-83. DOI: 10.1016/j. jdent.2016.11.002

10. Torres-de Araújo JR, Tomaz-de Lima RR, FerreiraBendassolli IM, Costa-de Lima K. Functional, nutritional and social factors associated with mobility limitations in the elderly: A systematic review. Salud Publica Mex. 2018;60(5):579-85. DOI: 10.21149/9075

11. Beelen J, Roos NM, Groot LCPGM. Protein enrichment of familiar foods as an innovative strategy to increase protein intake in institutionalized elderly. J Nutr Heal Aging. 2017;21(2):173-179. DOI: 10.1007/s12603-016-0733-y

12. Aajami Z, Kazazi L, Toroski M, Bahrami M, Borhaninejad V. Relationship between Depression and Cognitive Impairment among Elderly: A Cross-sectional Study. J Caring Sci.. 2020;9(3):143-53. DOI: $10.34172 /$ jcs.2020.022

13. Wang J, Mann F, Lloyd-Evans B, Ma R, Johnson S. Associations between loneliness and perceived social support and outcomes of mental health problems: A systematic review. BMC Psychiatry. 2018;18(1):1-16. DOI: 10.1186/s12888-018-1736-5

14. Carneiro JA, Ramos GCF, Barbosa ATF, Medeiros SM, Almeida Lima C, Da Costa FM, et al. Prevalence and factors associated with polypharmacy in community elderly: Population based epidemiological study. Med. 2018;51(4):254-64. DOI: 10.11606/issn.2176-7262. v51i4p254-264

15. Gatto CM, Jorge MSG, Wibelinger LM, Bertolin TE, Portella MR, Doring M. Prevalência de polifarmácia, benzodiazepínicos e fatores associados em idosos institucionalizados. Rev Bras Ciências do Envelhec Hum. 2019;16(3):47-58. DOI: 10.5335/rbceh.v16i3.7797

16. Rodrigues MCS, Oliveira C. Interações medicamentosas e reações adversas a medicamentos em polifarmácia em idosos: Uma revisão integrativa. Rev Lat Am Enfermagem. 2016;24. DOI: 10.1590/1518-8345.1316.2800

17. PORTUGAL. Ministério da Solidariedade e da Segurança Social. Portaria n.o 67/2012 de 21 de março. Diário da República. 2012;58(1):1324-9.

18. WHO. The Pursuit of Responsible Use of Medicines: Sharing and Learning from Country Experiences. World Health Organazation. 2012. Available from: https://www. who.int/activities/promoting-rational-use-of-medicines.

19. PORTUGAL. Ministério da Saúde. Decreto-lei n.o 176/2006, de 30 de Agosto. Diário da República, 1a série. 2006;167:6297-6383. 
20. PORTUGAL. Ministério da Saúde. Decreto-lei no 176/2006, de 30 de agosto. Diário da República, 1a série. 2006;167:6297-6383.

21. PORTUGAL. Presidência do Conselho de Ministros. Decreto-Lei no 112/2019, de 16 de agosto. Diário da República, 1a série. 2019;156:49-60.

22. WHO. Medication Without Harm [. World Health Organization. 2017. Available from: http://apps.who. int/iris/bitstream/10665/255263/1/WHO-HIS-SDS2017.6-eng.pdf?ua=1\&ua=1.

23. PORTUGAL. Ministério da Saúde. Decreto-Lei n.o 307/2007, de 31 de Agosto - Regime jurídico das farmácias de oficina. Diário da República, 1a série. 2007;(168):6083-91.

24. PORTUGAL. Ministério da Saúde. Portaria no 1427/2007, de 2 de novembro. Diário da República, 1a série. 2007;211:7991-2.

25. PORTUGAL. Ministério da Saúde.. Portaria no 1429/2007, de 2 de novembro. Diário da República, 1a série. 2007;211:7993.

26. Faller EM, Hernandez MT, Hernandez AM, Risia J, Gabriel S. Emerging Roles of Pharmacist in Global Health : An Exploratory Study on their Knowledge , Perception and Competency. Arch Pharm Pract. 2020;11(1):40-46.

27. Scott MA, Heck JE, Wilson CG. The Integral Role of the Clinical Pharmacist Practitioner in Primary Care. N C Med J. 2017;78(3):181-185. DOI: 10.18043/ncm.78.3.181

28. Taylor S, Cairns A, Glass B. Consumer perspectives of expanded practice in rural community pharmacy. Res Soc Adm Pharm [Internet]. 2021;17(2):362-367. DOI: 10.1016/j.sapharm.2020.03.022

29. Pita JR, Bell V. A farmácia em Portugal nos últimos 30 anos. Algumas reflexões sobre a farmácia de oficina ou comunitária. Debater a Eur. 2016;15:197-215. DOI: $10.14195 / 1647-6336 \quad 15 \quad 11$

30. Pita JR. Deontologia Farmacêutica. In: Guerreiro MP, Fernandes AA, editors. Deontologia e Legislação Farmacêutica. Lisboa: Lidel-Edições Técnicas, Lda; 2013. p. 17-28.

31. PORTUGAL. Asembleia da República. Lei n.o 131/2015 de 4 de setembro. Diário da República 1a série. 2015; $173: 7010-48$

32. Blouin RA, Adams ML. The Role of the Pharmacist in Health Care. N C Med J. 2017;78(3):165-167. DOI: 10.18043/ncm.78.3.165

33. Handyside L, Warren R, Devine S, Drovandi A. Utilisation of the PRECEDE-PROCEED model in community pharmacy for health needs assessment: A narrative review. Res Soc Adm Pharm [Internet]. 2021;17(2):292-299. DOI: 10.1016/j.sapharm.2020.03.021
34. PORTUGAL. Ministério do Trabalho S e SS (MTSSS). Carta Social - Rede de serviços e equipamentos - Relatório 2018. Gabinete de Estratégia e Planeamento. Lisboa; 2019. Available from: http://www.cartasocial.pt/pdf/csocial2018. pdf.

35. PORTUGAL. GEP - Gabinete de Estratégia e Planeamento. Rede de Serviços e Equipamentos. http://www.cartasocial. pt/index2.php. 2020.

36. Farias IPS, Montenegro LAS, Wanderley RL, Pontes JCX de, Pereira AC, Almeida LFD, et al. Physical, nutritional and psychological states interfere with health related quality of life of institutionalized elderly. BMC Geriatr. 2020;20:1-10. DOI: 10.1186/s12877-020-01791-6

37. Kosari S, McDerby N, Thomas J, Naunton M. Quality use of medicines in aged care facilities: A need for new models of care. J Clin Pharm Ther. 2018;43(4):591-593. DOI: 10.1111/ jcpt.12714

38. Almeida CV, Silva CR, Rosado D, Miranda D, Oliveira D, Mata F, et al. Manual De Boas Práticas Literacia Em Saúde.. Direção-Geral da Saúde. 2019. 1-59 p. DOI: 10.13140/ RG.2.2.17763.30243

39. PORTUGAL. Ministério da Saúde. Decreto-Lei n.o 307/2007, de 31 de Agosto. 2007;1-19.

40. Pepe GM, Kaefer TN, Goode JV “Kelly” R. Impact of pharmacist identification of medication-related problems in a nontraditional long-term care pharmacy. J Am Pharm Assoc 2018;58(4):S51-4. DOI: 10.1016/j. japh.2018.04.026

41. PORTUGAL. Comissão setorial para a saúde. Gestão da Medicação nas Estruturas Residenciais para Pessoas Idosas (ERPI) [Internet]. Lisboa; 2014. Available from: http://www1.ipq.pt/PT/SPQ/ComissoesSectoriais/CS09/ Documents/Recomendacao_paraGT_ERPI.pdf

42. Koprivnik S, Albiñana-Pérez MS, López-Sandomingo L, Taboada-López RJ R-PI. Improving patient safety through a pharmacist-led medication reconciliation programme in nursing homes for the elderly in Spain. Int J Clin Pharm. 2020;42(2):805-812. DOI: 10.1007/ s11096-020-00968-8

43. Litsey J. Evolution of Consulting Pharmacy and Medication Management. Top Geriatr Med Med Dir. 2015;37(1):1-5.

44. Barnett N. "Consultant pharmacist" - What does it mean? Hosp Pharm. 2008;15(2):34.

45. Malson $\mathrm{G}$. The role of the consultant pharmacist in the NHS. Clin Pharm. 2015;7(9):1-6. DOI: 10.1211/ CP.2015.20069457

46. Lewis R, Mortimore G. Role of the consultant pharmacist in clinical practice. Prescriber. 2018;29(8):19-22. DOI: 10.1002/psb.1695

47. Disalvo D, Luckett T, Bennett A, Davidson P, Agar M. Pharmacists' perspectives on medication reviews for 
long-term care residents with advanced dementia: a qualitative study. Int J Clin Pharm. 2019;41(4):950-962. DOI: 10.1007/s11096-019-00821-7

48. O’Donnell D, Beaton C, Liang J, Basu K, Hum M, Propp A, Yanni L, Chen Y GPC. Impact of a Pharmacist-Driven Medication Reconciliation Program during Transitions to Long-Term Care and Retirement Homes. Healthc Q. . 2020;23(3):34 40. DOI: 10.12927/hcq.2020.26335

49. Litsey J. Evolution of Consulting Pharmacy and Medication Management. Top Geriatr Med Med Dir. 2015;37(1):1-5.

50. Thomas J. Pharmacy organazations. In: Smith MI, Wertheimer AI, Fincham JE, editors. Pharmacy and the US Health Care System. Phamaceutical Press; 2013.

51. UK. Department of Health. A Vision for Pharmacy in the New NHS 2003. Available from: http://data.parliament. uk/DepositedPapers/Files/DEP2009-0485/DEP20090485.pdf.
52. Lee SWH, Mak VSL, Tang YW. Pharmacist services in nursing homes: A systematic review and meta-analysis. $\mathrm{Br}$ J Clin Pharmacol. 2019;85(12):2668-2688. DOI: 10.1111/ bcp. 14101

53. DH/MPIG. Guidance for the Development of Consultant Pharmacist Posts. 2005. Available from: http://www.codeg. org/fileadmin/codeg/pdf/DH_cons_pharm.pdf.

54. UK. Consultant Pharmacists Short Life Working Group. Consultant Pharmacist Guidance. 2020. Available from: https:/www.hee.nhs.uk/sites/default/files/documents/ Consultant Pharmacist Guidance Final Jan2020.pdf.

55. The Lewis Group. CMS Review of Current Standards of Practice for Long-Term Care Pharmacy Services Long-Term Care Pharmacy Primer. 2004. Available from: https://www.cms.gov/Research-Statistics-Dataand-Systems/Statistics-Trends-and-Reports/Reports/ Downloads/LewinGroup.pdf. 